UCLA $/ 99 /$ TEP $/ 25$

INLO-PUB-11/99

hep-lat/9905029

\title{
On P-vortices and the Gribov problem
}

\author{
Tamás G. Kovácsi \\ Instituut-Lorentz for Theoretical Physics, P.O.Box 9506, 2300 RA, Leiden, The \\ Netherlands \\ e-mail: kovacs@lorentz.leidenuniv.nl \\ and \\ E. T. Tomboulisf \\ Department of Physics, UCLA, Los Angeles, CA 90095-1547 \\ e-mail: tombouli@physics.ucla.edu
}

\begin{abstract}
We study the possible connection between centre vortices and P-vortices in $\mathrm{SU}(2)$ gauge theory. After briefly recalling some essential properties of centre vortices we point out that there is no known a priori connection between the gauge dependent $\mathrm{P}$-vortices and the gauge invariant centre vortices. We then show by Monte Carlo simulations that the 'centre projected physics' strongly depends on the gauge copy from which the maximal centre gauge fixing is started. This reveals the presence of Gribov problems, and casts some doubts on the physical meaning of P-vortices, and should be further investigated.
\end{abstract}

\footnotetext{
${ }^{1}$ Research supported by FOM.

${ }^{2}$ Research supported by NSF grant nsf-phy 9531023
} 
Recently there has been considerable interest in the role that vortices might play in confinement in non-Abelian gauge theories [1]. One approach towards isolating such configurations in the vacuum which has been pursued by several authors [2] - [5], [7] is that of the so called centre projection and projection vortices. Centre projection involves a gauge fixing to the so called maximal centre gauge (MCG) that puts the $\mathrm{SU}(\mathrm{N})$ gauge group valued link variables as close as possible to elements of the centre, $\mathrm{Z}(\mathrm{N})$. After gauge fixing the link variables are 'projected' onto the centre, i.e. they are replaced by the closest centre element. This procedure is in complete analogy with Abelian projection in the maximal Abelian gauge. Most of the results obtained with MCG so far are for the case of $N=2$ and we shall also mostly concentrate on this case.

The excitations of the resulting $\mathrm{Z}(2)$ gauge theory after the projection are very simple objects: co-closed (their co-boundary is empty) sets of plaquettes each carrying -1 flux. In analogy with vortices in the full $\mathrm{SU}(2)$ theory, these are referred to as projection vortices or P-vortices. A detailed study of full SU(2) Wilson loops with an even and odd number of $\mathrm{P}$-vortices linking with them showed that the sign of large Wilson loops is strongly correlated with the parity of the number of $\mathrm{P}$-vortices linking with the loop [2]. Although no exact connection is known between P-vortices and vortices in the full $\mathrm{SU}(2)$ system, this property led to the tentative identification of P-vortices with the 'cores' of full SU(2) vortices. The centre projected configurations were found in [2] to carry the full $\mathrm{SU}(2)$ string tension, and, furthermore, the density of P-vortices was claimed to be a scaling physical quantity [3, 2]. Further work in MCG and centre projection led to suggestions that P-vortices account for the finite temperature properies of the $\mathrm{SU}(2)$ theory [4], and that the elimination of P-vortices results in the loss of confinement and chiral symmetry breaking [5].

In this letter we would like to point out some potential problems with this picture and draw attention to some questions that in our view would have to be clarified. We first point out that, although P-vortices are known in some cases to be correlated with centre fluctuations of Wilson loops, there is in fact no established connection between topological centre vortices in $S U(N)$ gauge theory and P-vortices. We then proceed to demonstrate some potential problems with the gauge fixing and projection procedure that are due to the Gribov ambiguity. By presenting results of a Monte Carlo computation, we show that if the centre gauge fixing is started from already Lorentz gauge fixed configurations then on the average the MCG fixing arrives to a higher local maximum of the gauge fixing functional than when started from a random gauge; but at the same time the projected configurations have only a very small fraction of the full $\mathrm{SU}(2)$ string tension. Moreover, the density of P-vortices which is claimed to be a scaling physical quantity, is significantly smaller than without an initial Lorentz gauge fixing. This, when combined with the previously known fact that centre projection results are unstable under local smoothing [2], casts doubts on the physical relevance of P-vortices and calls for further investigations on whether P-vortices can be defined in an unambiguous manner, so that the projection-physics be independent of the details 
of the gauge fixing procedure.

Centre vortices versus P-vortices A vortex is a configuration of the gauge potentials $\mathbf{A}_{\mu}$ topologically characterised by nontrivial elements of $\pi_{1}(S U(N) / Z(N))=Z(N)$. This means that over a sufficiently large loop the configuration is characterised by a singular gauge transformation which cannot be consistently defined throughout the space encircled by the loop without encountering a topological obstruction; the multivaluedness ambiguity is by elements of $Z(N)$. Note that the topological $Z(N)$ flux is conserved only mod $N$, so the number of vortices linked with a given loop is defined only $\bmod N$. On the lattice only configurations with one-plaquette action near its maximum contribute significantly at large $\beta$. In fact such local smoothness is essential in order to be able to talk of vortices relevant to the continuum limit in the first place; according to rigorous theorems, only for lattice configurations with sufficiently small plaquette function variations from the maximum is it possible to unambiguously define a continuum interpolation assignable to a topological sector. This implies that only locally smooth very extended vortices ('thick' vortices) are of interest. They incorporate potential long distance disordering together with UV asymptotic freedom. It was indeed found [6] that the induced centre fluctuations for large Wilson loops in $S U(2)$ and $S U(3)$ carry the full string tension, and in a manner which is perfectly stable under repeated smoothings removing short distance fluctuations.

Now given such an $S U(2)$ vortex configuration, how can a P-vortex be 'associated' with it? By considering regular gauge transforms of it, the nontrivial $Z(2)$ element of the singular gauge transformation asymptotically characterising the vortex may be made to be distributed over many or few links of an encircling loop. Assume that by a gauge transformation it is concentrated on just one. One may then try to extend this gauge transformation in directions perpendicular to the loop so that a jump by -1 occurs when crossing this 'wall'. In essence one is trying to compress the thick vortex into a thin one by a gauge transformation. In the presence of the lattice cutoff, in the corresponding $\mathrm{Z}(2)$ projected configuration this may appear as a $Z(2)$ vortex (P-vortex) linking with the loop. However, it is clear that this procedure is ambiguous. A slightly different gauge transformation may distribute the $Z(2)$ jump over two or three links, in such a way that the $Z(2)$ projected configuration has one, three, or much worse, zero or two P-vortices linking with the loop. There is no guarantee that a gauge condition such as the MCG will always pick out one but not the other from two such configurations. Or that starting from gauges where the vorticity along a large loop is spread out as smoothly as possible, Gribov problems will not arise in trying to go over to centre gauges. At any rate, we are not aware of any well-defined procedure of associating the gauge-dependent concept of a P-vortex with the notion of an extended $\pi_{1}(S O(3))$ vortex in the gauge field.

All this raises the possibility that variations in the centre gauge fixing procedure and Gribov ambiguities may lead to widely different number of P-vortices. Worse, there may be no stable mod 2 correspondence between the number of P-vortices and the number of $S U(2)$ vortices. The string tension from P-vortices may then change 
substantially under similar gauge fixing schemes. We next proceed to investigate some of these possible problems.

The Gribov problem The most essential ingredient in the definition of P-vortices is the gauge fixing to the maximal centre gauge (MCG) by maximizing the functional

$$
f[U]=\sum_{l}\left(\operatorname{tr} U_{l}\right)^{2}
$$

where the summation is over all the links of the lattice. After gauge fixing, a "projection" is performed which amounts to the replacement of all the link variables with the closest centre element. In the case of $\mathrm{SU}(2)$ this is simply a $U_{l} \rightarrow \operatorname{sign} \operatorname{tr} U_{l}$ replacement which results in a $\mathrm{Z}(2)$ gauge configuration. The procedure is completely analogous to Abelian projection in the maximal Abelian gauge.

Unfortunately, in practice the gauge fixing is ambiguous because the gauge fixing functional $f$ has several local maxima over the whole gauge orbit. It is practically impossible to select the absolute maximum of these local maxima, instead, a local maximum is achieved only. Gauge non-invariant quantities can depend on which local maximum is selected. A typical way of dealing with this problem is to perform the gauge fixing on several randomly selected gauge copies of the same configuration and using only the one for which the highest maximum of $f$ is obtained [2]. In the present paper we would like to study how sensitive the "centre projected physics" is to the Gribov ambiguity.

Physically, the MCG tries to compress most of the fluctuations into objects on the one lattice spacing scale, i.e. P-vortices. On the other hand Lorentz gauge achieves exactly the opposite. By putting all the links as close to the identity as possible, it tries to spread the fluctuations evenly. The question we would like to ask is whether there is a significant difference in the performance of the MCG fixing depending on what type of gauge copy we start it from. Therefore we made two gauge copies of the same set of configurations, one random copy and another fixed to the Lorentz gauge. We then compared the projection physics of these two equivalent ensembles.

We used a set of $10012^{4} \mathrm{SU}(2)$ gauge configurations generated with the Wilson action at $\beta=2.4$. The random gauge copies were simply the ones resulting from the Monte Carlo and the other ensemble was prepared by fixing the same set of configurations to the Lorentz gauge by maximising $\sum_{l} \operatorname{tr} U_{l}$. We then performed MCG fixing and projection on both ensembles using the over-relaxation algorithm described in [2]. The iteration was stopped when the change in gauge fixing action per degree of freedom was smaller than $1.0 \times 10^{-9}$.

In Figure 1 we compare the heavy quark potential obtained from the two centre projected ensembles as well as the $\mathrm{SU}(2)$ configurations. As expected from the work of Del Debbio et al. [2], the projected ensemble for which the gauge fixing was started from a random gauge, reproduces the full $\mathrm{SU}(2)$ string tension but without the coulomb term in the potential. On the other hand, if the MCG fixing is started from already Lorentz 


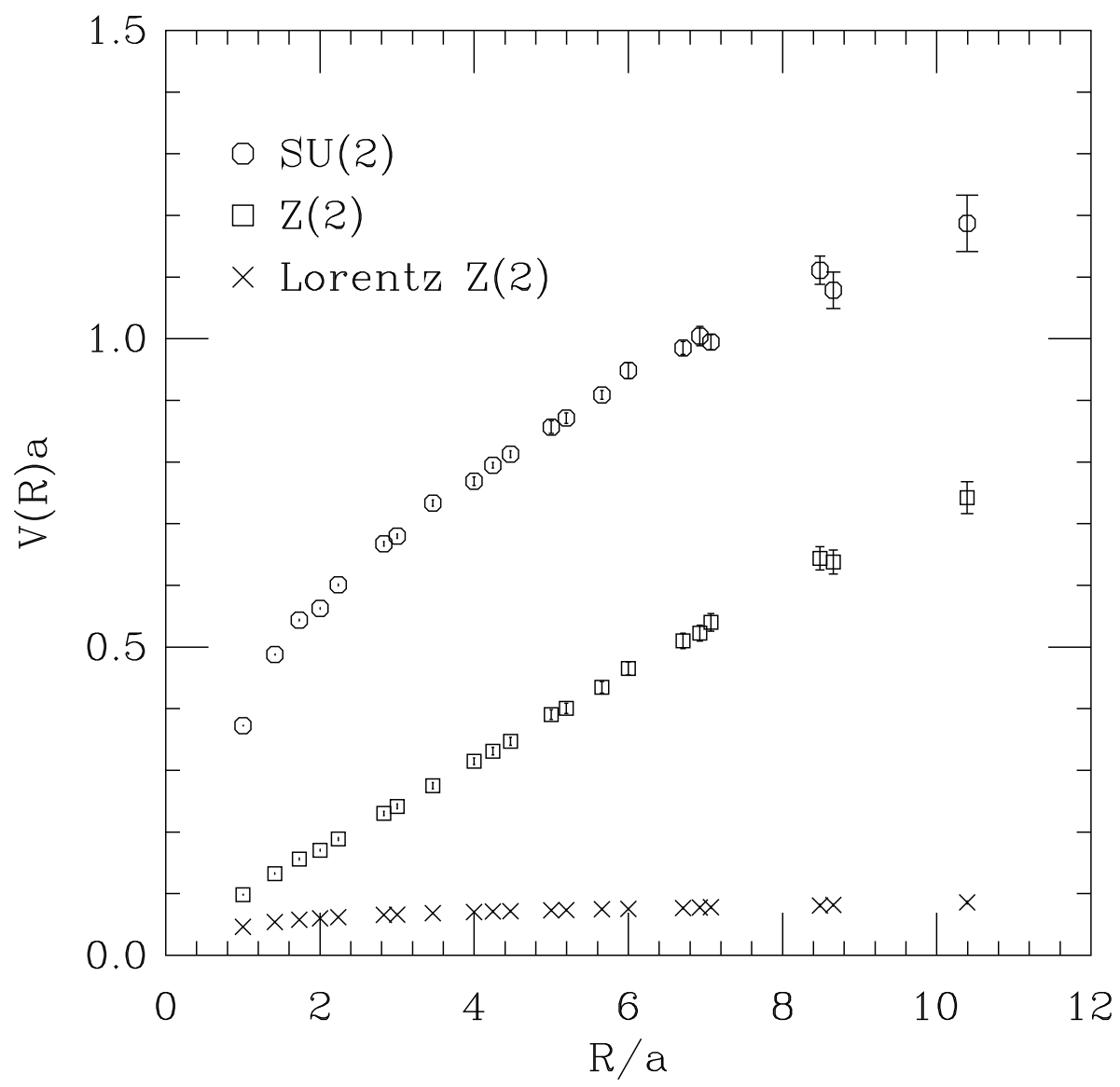

Figure 1: The heavy quark potential measured on a set of $10012^{4}$ Wilson $\beta=2.4$ gauge configurations. $\mathrm{SU}(2)$ is the full $\mathrm{SU}(2)$ potential, $\mathrm{Z}(2)$ is measured on the centre projected configurations, and "Lorentz Z $(2)$ " is measured on the first Lorentz gauge fixed then MCG fixed and then centre projected ensemble.

gauge fixed configurations, the resulting "projection physics" is entirely different. The string tension is compatible with zero, there is no confinement. We stress that the two projected ensembles producing so vastly different projection physics, should in principle be exactly equivalent, the two $\mathrm{SU}(2)$ ensembles being gauge copies of one another.

It is also instructive to compare the average value of the maximum of the gauge fixing function obtained for the two ensembles. In the random gauge started ensemble it is 3.0914(10), while in the Lorentz gauge started ensemble it is 3.1012(8). In fact, the MCG fixing arrives at a higher local maximum if started from Lorentz gauge and 
not a random gauge.

It has been observed that the area-density of $\mathrm{P}$-vortices is a properly scaling physical quantity [2]. This quantity is essentially the volume density of -1 plaquettes in the projected configurations, i.e., in lattice units

$$
p=\frac{N_{\text {vor }}}{6 \times \text { Volume }}
$$

where $N_{\text {vor }}$ is the number of -1 plaquettes. In a recent work the geometrical structure of these vortex surfaces on the dual lattice has been studied in great detail [7]. We also measured the P-vortex density in both ensembles. In the random gauge started ensemble we obtained $p=0.0552(5)$, consistently with Ref. [2]. The Lorentz gauge started ensemble produced a significantly smaller result, $p=0.0338(2)$. Considering the complete absence of a string tension in the latter ensemble, a drop of only about $40 \%$ in the vortex density indicates that the structure of these remaining P-vortices must be vastly different from the ones occurring in the random gauge started ensemble. They must be small localized vortices, not contributing to the string tension.

For comparison we repeated the above experiment using the same set of $\mathrm{SU}(2)$ gauge configurations but now instead of centre projecting in the MCG, we used Abelian projection in the maximal Abelian gauge. In this case the Gribov problem is also potentially present. The maximum of the gauge fixing action however shows only a slight difference if any between the two ensembles. We obtained 1.4620(4) for the ensemble started from a random gauge and 1.4632(4) for the Lorentz gauge started ensemble. Similarly, the heavy quark potentials, shown in Fig. 2 are also not as vastly different as in the case of the MCG. In fact, more extensive computations would be needed here in order to draw a definitive conclusion about the possible difference in the two string tensions. This indicates that the Gribov ambiguity probably does not influence the physical observables so severely in the MAG case as it does in the case of the MCG.

Some time ago it was noticed that a slight local smoothing of the SU(2) configurations decreases the centre projected string tension considerably [2]. This shows that $\mathrm{SU}(2)$ configurations with similar long distance properties but with different short distance fluctuations, can produce different projection physics. Somehow the centre projection entangles excitations living on different length scales and it is not clear whether the centre projected configurations really reflect the long distance $\mathrm{SU}(2)$ physics. Our present result is even more alarming. It shows that exactly gauge equivalent $\mathrm{SU}(2)$ configurations, when fixed to the MCG and centre projected, can produce $\mathrm{Z}(2)$ configurations with vastly different physical properties. This casts doubts on the physical meaning of P-vortices and certainly calls for a more detailed study of the Gribov problem in the context of centre projection. 


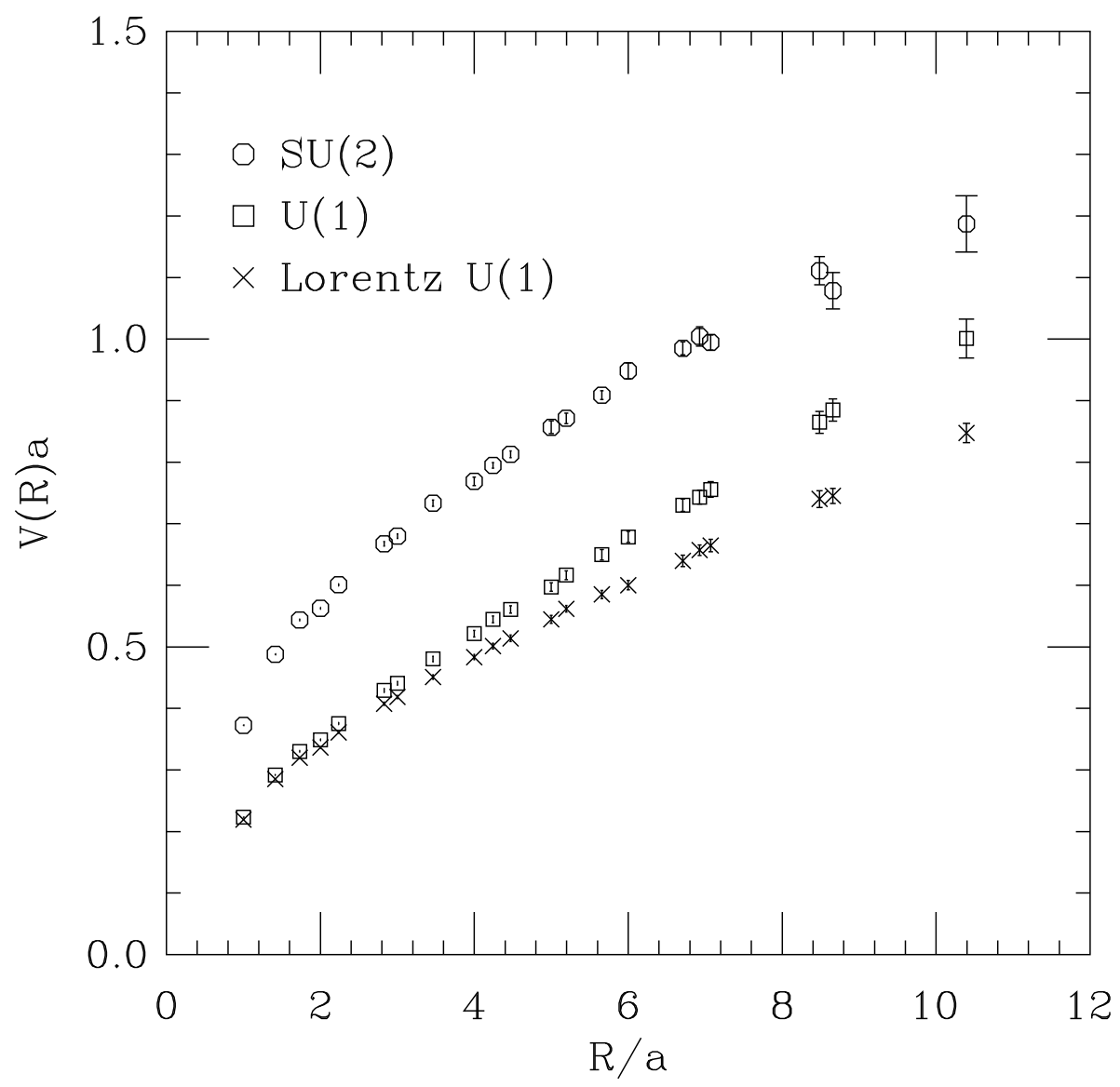

Figure 2: The heavy quark potential measured on a set of $10012^{4}$ Wilson $\beta=2.4$ gauge configurations. $\mathrm{SU}(2)$ is the full $\mathrm{SU}(2)$ potential, $\mathrm{U}(1)$ is measured on the Abelian projected configurations, and "Lorentz U(1)" is measured on the first Lorentz gauge fixed then maximal Abelian gauge fixed and then Abelian projected ensemble.

\section{Acknowledgements}

TGK thanks Pierre van Baal and Margarita García Pérez for discussions and Philippe de Forcrand for helpful correspondence.

\section{References}


[1] See e.g. the following contributions to Lattice '98, Boulder, CO: J. Greensite, M. Faber, and S. Olejník, hep-lat/9809053; C. Hoelbing, C. Rebbi, and V.A. Rubakov, hep-lat/9809113; J.D. Stack, and W. Tucker, hep-lat/9810011; P.W. Stephenson, hep-lat/9809127; T.G. Kovacs, and E.T. Tomboulis, heplat/9808046.

[2] L. Del Debbio, M. Faber, J. Giedt, J. Greensite, and Š. Olejník, Phys. Rev. D58 (1998) 094501.

[3] K. Langfeld, H. Reinhardt, and O. Tennert, Phys. Lett. B419 (1998) 317.

[4] M. Engelhardt, K. Langfeld, H. Reinhardt, and O. Tennert, hep-lat/9904004.

[5] Ph. de Forcrand, and M. D'Elia, hep-lat/9901020.

[6] T. G. Kovács, E. T. Tomboulis, Phys. Rev. D57 (1998) 4054; Phys. Lett. B443 (1998) 239.

[7] R. Bertle, M. Faber, J. Greensite, and Š. Olejník, JHEP 9903 (1999) 019. 